\title{
Kondo Proximity Effect: How Does a Metal Penetrate into a Mott Insulator?
}

\author{
R. W. Helmes, ${ }^{1}$ T. A. Costi, ${ }^{2}$ and A. Rosch ${ }^{1}$ \\ ${ }^{1}$ Institute for Theoretical Physics, University of Cologne, 50937 Cologne, Germany \\ ${ }^{2}$ Institute of Solid State Research, Research Centre Jülich, 52425 Jülich, Germany
}

(Received 7 May 2008; published 6 August 2008)

\begin{abstract}
We consider a heterostructure of a metal and a paramagnetic Mott insulator using an adaptation of dynamical mean-field theory to describe inhomogeneous systems. The metal can penetrate into the insulator via the Kondo effect. We investigate the scaling properties of the metal-insulator interface close to the critical point of the Mott insulator. At criticality, the quasiparticle weight decays as $1 / x^{2}$ with distance $x$ from the metal within our mean-field theory. Our numerical results (using the numerical renormalization group as an impurity solver) show that the prefactor of this power law is extremely small.
\end{abstract}

DOI: 10.1103/PhysRevLett.101.066802

Introduction. - In the last few years, an enormous amount of interest has arisen in heterostructures fabricated out of strongly correlated materials. Driven by the prospect of new effects and devices based on correlated electron compounds, a wide range of systems has been studied experimentally and theoretically. For example, the interface of two Mott insulators can show metallic behavior [15] or can even become superconducting [6]. The conducting layers can, for example, arise from interface charges induced by the Coulomb interactions. As shown by Thiel et al. [5], such interfaces can be manipulated by gate voltages thereby opening the prospect for interesting novel devices.

The physics of such inhomogeneous systems can also play a role for the properties of bulk materials where competing phases lead to the formation of domain walls. For example, it has been argued in Ref. [7] that the conductivity close to the endpoint of the first-order Mott transition in certain organic salts of the $\kappa$-ET family is dominated by domain wall effects. Also, for cold atoms, the trapping potential naturally makes the experimental systems inhomogeneous which often leads to coexisting phases and corresponding phase boundaries [8].

In this Letter, we will investigate the interface of a metal and a Mott insulator. How does a metal penetrate into a Mott insulator? The main difference between a Mott insulator and an ordinary band insulator is the presence of magnetic degrees of freedom arising from the localized spins. While the large charge gap, of the order of the local Coulomb repulsion $U$, prohibits tunneling of electrons into a Mott insulator, the resonant spin flip scattering opens a new channel for tunneling via the well-known Kondo effect and allows metallic behavior to be induced within the Mott insulator. Because of this "Kondo proximity effect," an insulating layer adjacent to the metal will also become metallic. In this manner the metal "eats" itself layer by layer into the Mott insulator if not stopped either by magnetism or thermal fluctuations. Here, we study this physics within the simplest setup consisting of a particlehole symmetric Hubbard model (see below) where the local interaction $U$ jumps across the interface from $U=$
PACS numbers: 73.20. $-\mathrm{r}, 71.27 .+\mathrm{a}, 71.30 .+\mathrm{h}$

$U_{\text {left }} \ll U_{c}$ to a value $U=U_{\text {right }}$ close to the critical coupling $U_{c}$ of the Mott transition. For such a model, the charge is always homogeneous, and no complications due to charge-reconstruction or charged interface layers arises. Furthermore, we only consider a paramagnetic Mott insulator and comment on the role of magnetism only in the conclusions.

For one-dimensional systems, powerful numerical methods like density matrix renormalization group are available to study inhomogeneous strongly interacting models [9]. For three-dimensional systems, however, further approximations are necessary. Here, the method of choice to study the Mott transition is the so-called dynamical mean-field theory (DMFT) [10,11]. Within DMFT, the only approximation is to neglect nonlocal contributions to the selfenergy. This approximation can be used both for homogeneous $[10,11]$ and inhomogeneous $[3,12-15]$ problems. In the case of a heterostructure, each layer is effectively mapped to a single-impurity Anderson model. These are coupled by a self-consistency condition, see below.

Potthoff and Nolting used this spatially resolved DMFT to study the Mott transition at surfaces using the semiinfinite Hubbard model [16]. In this context, also the question was studied how a metallic surface influences the insulating bulk. Spatially resolved DMFT has also been applied $[1,3,14]$ to investigate heterostructures of Mott insulators and band insulators.

A main problem of DMFT is the need for a reliable and efficient method to solve the effective impurity problem. Previous applications of DMFT to inhomogeneous systems used impurity solvers based on exact diagonalization of small systems [16], a linearized version of DMFT close to the critical point [16], a two-site approximation [1,3] or slave-boson mean-field theory [17], implying severe further approximations, or started from simpler models such as the Falicov-Kimball model [14]. Only recently [8,18], the numerical renormalization group (NRG) method $[19,20]$ was implemented as an impurity solver to study the Mott transition of trapped atoms in an optical lattice. We will also use this approach here as the NRG appears to be the only method presently available which can quanti- 
tatively resolve quasiparticle weights as small as $10^{-3}$ which are needed to describe the physics close to the Mott transition.

After introducing the model and our method (DMFT for inhomogeneous systems + NRG), we will first investigate the heterostructure at finite $T$ and for $U_{\text {right }}=U_{c}$. We will then analyze the $T=0$ scaling properties of the interface region at $U_{\text {right }} \lesssim U_{c}$ and $U_{\text {right }} \gtrsim U_{c}$ using both DMFT + NRG and a Ginzburg-Landau type analysis.

Model and method. - To investigate the junction of the metal and the Mott insulator, we will consider the halffilled Hubbard model

$$
\mathcal{H}=-t \sum_{\langle i j\rangle, \sigma} c_{i \sigma}^{\dagger} c_{j \sigma}+\sum_{i} U_{i}\left(n_{i \uparrow}-\frac{1}{2}\right)\left(n_{i \downarrow}-\frac{1}{2}\right)
$$

on a three-dimensional cubic lattice with the half band width $D=6 t$. While we will consider a uniform hopping $t$, we choose $U_{i}=U_{\text {left }}=D$ for $x \leq 0$ describing a metal with a sizable quasiparticle weight $Z_{\text {metal }}=0.62$. For sites with $x \geq 1$, we use an interaction $U_{i}=U_{\text {right }} \sim U_{c}$ close to the critical value, $U_{c} \approx(2.79 \pm 0.01) D$ which separates the metallic from the insulating phase in the bulk. Note that the system is translationally invariant in the $y z$ directions as $U_{i}$ is constant within each $y z$ layer.

The DMFT algorithm for this heterostructure is almost identical to the standard one [10]. As all sites within a single $y z$ layer are equivalent, it is sufficient to solve only one effective Anderson impurity problem (using NRG $[20,21])$ for each $y z$ layer to obtain an $x$ dependent selfenergy $\Sigma_{x}(\omega)$. From this, one obtains the lattice Greens function

$$
\hat{G}^{\text {lat }}\left(\epsilon_{\mathbf{k}_{\perp}}, \omega\right)=\left[\omega-\epsilon_{\mathbf{k}_{\perp}}-t_{x x^{\prime}}-\delta_{x x^{\prime}} \Sigma_{x}(\omega)\right]^{-1}
$$

written as a matrix in the $x$ coordinates where $t_{x x^{\prime}}=t$ for $x^{\prime}=x \pm 1$ and 0 otherwise and $\epsilon_{\mathbf{k}_{\perp}}$ is the dispersion within each layer. From $\hat{G}^{\text {lat }}$, one determines the local Greens function which is used [10] to derive a new effective Anderson impurity model with the Greens function $G_{\text {imp }}(x)$ for each layer using the self-consistency equation

$$
\left.G_{x}^{\mathrm{imp}}(\omega) \stackrel{!}{=} \int d \epsilon_{\mathbf{k}_{\perp}} N_{2 d}\left(\epsilon_{\mathbf{k}_{\perp}}\right) \hat{G}^{\mathrm{lat}}\left(\epsilon_{\mathbf{k}_{\perp}}, \omega\right)\right|_{x x}
$$

where $N_{2 d}(\epsilon)=\sum_{\mathbf{k}_{\perp}} \delta\left(\epsilon-\epsilon_{\mathbf{k}_{\perp}}\right)$ is the two-dimensional density of states of the $y z$ layers. To avoid numerical difficulties associated with the logarithmic divergence of $2 d$ cubic density of states, we use $N_{2 d}(\epsilon)=1 /(8 t)$ for $|\epsilon|<4 t$. This does not affect any universal properties discussed below and leads only to small changes in $U_{c}$ (and other numerical prefactors) of about 10\%. From Eq. (2), it seems that one has to invert a matrix for each value of $\omega$ and $\epsilon_{\mathbf{k}_{\perp}}$. Fortunately, this computationally expensive step can be simplified by diagonalizing $\hat{G}^{\text {lat }}(0, \omega)^{-1}$ using the orthogonal matrix $\hat{O}(\omega)$, $\hat{O}^{T}(\omega) \hat{G}^{\mathrm{lat}}(0, \omega)^{-1} \hat{O}(\omega)=\hat{M}(\omega)$, so that $\hat{G}^{\mathrm{lat}}\left(\epsilon_{\mathbf{k}_{\perp}}, \omega\right)=$
$\hat{O}(\omega)\left[\hat{M}(\omega)-\epsilon_{\mathbf{k}_{\perp}}\right]^{-1} \hat{O}^{T}(\omega)$. We use 20 metallic layers with $U=D$ and 40 layers with $U \sim U_{c}$ which is sufficiently large to avoid any finite size effects.

Results. -Figure 1 shows the layer dependence of the spectral function for $U_{\text {right }}=U_{c}$ for $x \geq 0$ and for a low temperature $T=1.14 \times 10^{-5} \mathrm{D}$. The metallic side is only weakly affected by the presence of the insulator as within our paramagnetic, particle-hole symmetric model, no Friedel oscillations occur. All layers with $U=U_{c}$ show pronounced Hubbard bands. The width of the sharp quasiparticle peak, which describes the penetration of the metal into the quantum-critical Mott state, decays rapidly. The quasiparticle peak collapses completely (up to an exponentially small feature) from the 5th layer on, when the Kondo temperature of the corresponding impurity model becomes much smaller than $T$.

For a quantitative analysis of how the metal penetrates into the Mott insulator, we investigate a heterostructure consisting of a "good metal," $U_{\text {left }}=D$, and a "bad metal," $U_{\text {right }} \leqslant U_{c}$, at $T=0$. For $T=0$, the quasiparticle weight $Z_{x}$ of layer $x$ is well defined and can be obtained from $Z_{x}=\left[1-\partial_{\omega} \operatorname{Re} \Sigma_{x}(\omega)\right]^{-1}$. Figure 2 shows the quasiparticle weight $Z$ as a function of the distance $x$ from the interface. Upon increasing $U_{\text {right }}$, the quasiparticle weight deep in the bad metal decreases linearly with $U_{c}-U_{\text {right }}$ [10]. Close to the critical point, one expects scaling behavior, and indeed we observe in Fig. 3

$$
Z_{x} \approx \frac{0.008 \pm 0.002}{x^{1 / \nu}} f\left[x\left(\frac{U_{c}-U_{\text {right }}}{U_{c}}\right)^{\nu}\right]
$$

with $\nu=1 / 2$ where $f[u]$ is an universal scaling function with $f[0]=1$ and $f[u \rightarrow \infty] \approx(0.150 \pm 0.005) u^{2}$ for $U_{\text {right }} \lesssim U_{c}$. The observation that DMFT is characterized by the usual mean-field exponent $\nu=1 / 2$ and the $1 / x^{2}$ decay of the correlation function in the quantum-critical regime is one of the main results of this Letter. Defining the

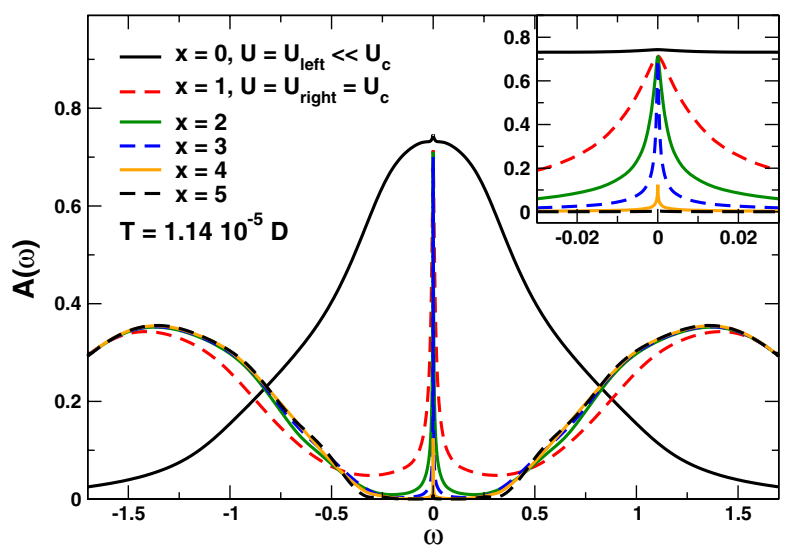

FIG. 1 (color online). Layer dependence of local spectral function close to the interface $x=0$ for $U_{\text {right }}=U_{c}$ and $T=$ $1.14 \times 10^{-5} D$. Inset: $A(\omega)$ near $\omega=0$. 


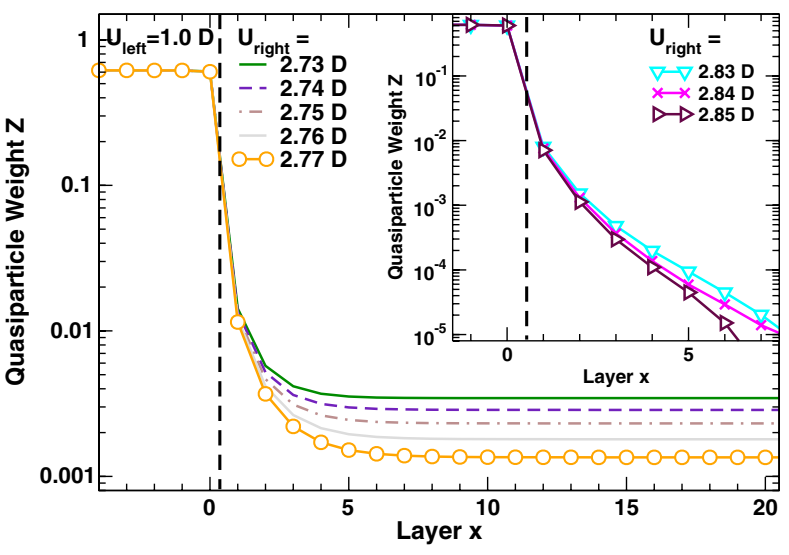

FIG. 2 (color online). Quasiparticle weight $Z$ for an inhomogeneous layered system describing a "good" metal $(U=$ $U_{\text {left }}=D$ for $\left.x<1\right)$ in contact with a "bad" metal $\left(U_{\text {right }} \lesssim\right.$ $U_{c}=(2.79 \pm 0.01) D$ for $\left.x \geq 1\right)$. Inset: Quasiparticle weight for a heterostructure of a good metal and a Mott insulator $\left(U_{\text {right }} \gtrsim\right.$ $U_{c}$ ). In both cases, $Z$ drops as $1 / x^{2}$ for $x<\xi$, see Fig. 3. For extremely small quasiparticle weights $Z<10^{-3}$, the numerical results eventually become unreliable.

correlation length $\xi$ by $f[u]=2$, we obtain

$$
\xi \approx 0.3\left(\frac{U_{c}}{U_{c}-U_{\text {right }}}\right)^{1 / 2} .
$$

In the Mott insulating phase at $T=0, U_{\text {right }} \gtrsim U_{c}$, the quasiparticle weight drops rapidly, see inset of Fig. 2. In this regime, the numerical calculations become difficult

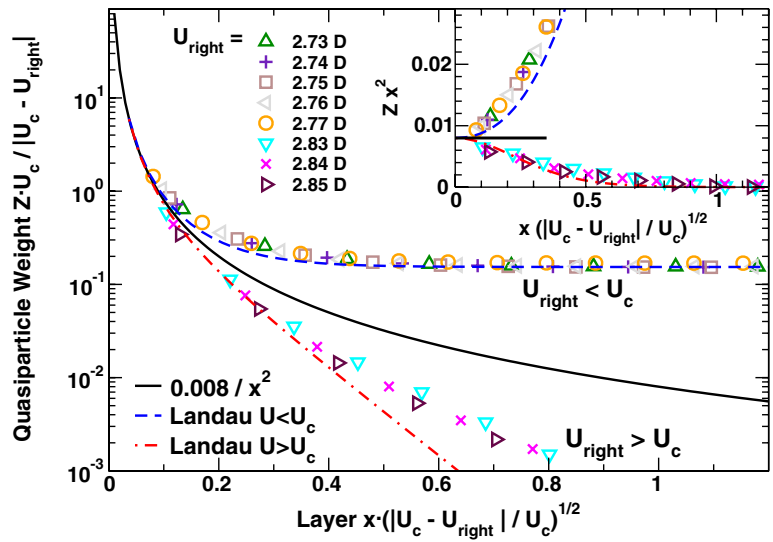

FIG. 3 (color online). Scaling plot [26] of the $T=0$ quasiparticle weight close to the quantum-critical point both for $U_{\text {right }}<$ $U_{c}$ (upper curves) and $U_{\text {right }}>U_{c}$ (lower curves) confirming $\nu=1 / 2$ in Eq. (4). In the quantum-critical regime $(x<\xi), Z \approx$ $0.008 / x^{2}$ (solid line). For $U_{\text {right }}<U_{c}, Z$ saturates at a finite value proportional to $U_{c}-U_{\text {right }}$ for $x>\xi$ while it drops exponentially for $U_{\text {right }}>U_{c}$, see Eq. (8). Dashed, dot-dashed lines: scaling curves obtained from a Ginzburg-Landau type analysis using Eqs. (6) and (7). The results for $Z U_{c} /\left(U_{\text {right }}-U_{c}\right)<0.05$ are not numerically reliable. and are unreliable for $Z<10^{-3}$. Nevertheless, in the regime where the numerical results are accurate, they confirm the scaling ansatz Eq. (4), as can be seen in detail in Fig. 3: In the quantum-critical regime, i.e., for $x<\xi$, the quasiparticle weight decays as $1 / x^{2}$ with the same prefactor as in Eq. (4). For $x>\xi$, however, $Z$ drops exponentially but remains always finite.

This picture is further corroborated by an analysis in the spirit of a Ginzburg-Landau mean-field treatment as in $[16,22,23]$. The basic idea is that close to $U_{c}$, the physics is mainly determined by the quasiparticle peak which can be characterized by a single number, the quasiparticle weight $Z_{x}$. We therefore approximate in Eq. (2) $\Sigma_{x}(\omega) \approx$ $\omega-\omega / Z_{x}$. The resulting local spectral function $-\frac{1}{\pi} \operatorname{Im} G_{x x}^{\text {lat }}(\omega)$ has a peak with a finite width. From this peak, one has to determine a single number describing the effective impurity model and from this, a new value of $Z_{x}$ using a Ginzburg-Landau expansion around the critical point.

$$
\begin{aligned}
& Z_{x}^{\prime}=\frac{3}{22}\left(Z_{x-1}+\frac{16}{3} Z_{x}+Z_{x+1}\right) \\
& Z_{x}=Z_{x}^{\prime}-\alpha \frac{U-U_{c}}{U_{c}} Z_{x}^{\prime}-\beta Z_{x}^{\prime 2}
\end{aligned}
$$

For the first step, Eq. (6), we have used the procedure described by Bulla, Potthoff, and Nolting [16,22] with $\int N_{2 d}(\epsilon) d \epsilon=16 t^{2} / 3$. For the homogeneous system with $U<U_{c}$, one obtains $Z=\frac{\alpha}{\beta} \frac{U_{c}-U}{U_{c}}$. Analyzing the asymptotic solutions of Eqs. (6) and (7) for the interface with $U_{\text {right }}=U_{c}$, we find $\lim _{x \rightarrow \infty} Z_{x}=9 /\left(11 \beta x^{2}\right)$. The model (6) and (7) reproduces the critical exponents of our DMFT calculation (4). Fitting the asymptotic formulas to the NRG results, we obtain $\alpha=15.7 \pm 5$ and $\beta=102 \pm 30$, reflecting the small prefactor in Eq. (4). While Potthoff and Nolting [16] have analyzed different critical exponents, their results are qualitatively fully consistent with ours.

In Fig. 3, we show that the Ginzburg-Landau analysis reproduces the DMFT scaling curves quite well as the parameters in (6) and (7) have been determined to fit the prefactor of the $1 / x^{2}$ law and the large $x$ limit for $U_{\text {right }}<$ $U_{c}$. The large deviations for $U_{\text {right }}>U_{c}$ arise in the aforementioned regime where the numerical calculations are no longer reliable. There is also a smaller deviation for $U_{\text {right }}<U_{c}$ which could be an indication that DMFT cannot be reduced to mean-field theory for a scalar as in (6) and (7) even in the scaling limit [24]. From the model (6) and (7), one can also extract the asymptotic behavior for large $x>\xi$

$$
Z_{x} \approx \frac{0.8 U_{c}}{U_{\text {right }}-U_{c}} \exp \left[-\frac{x}{\xi}\right] \text { for } x \rightarrow \infty
$$

with 


$$
\xi=\sqrt{\frac{3}{22 \alpha}}\left(\frac{U_{c}}{U_{\text {right }}-U_{c}}\right)^{1 / 2} \approx 0.09\left(\frac{U_{c}}{U_{\text {right }}-U_{c}}\right)^{1 / 2} .
$$

Regional Computing Center Cologne and financial support by the SFB 608 of the DFG.

Conclusions. - In this Letter, we have studied how a metallic state penetrates into a paramagnetic Mott insulator (or a bad metal). Using a scaling analysis close to the quantum-critical point, we have determined within dynamical mean-field theory the critical exponents and the asymptotic behavior of the quasiparticle weight close to and far away from the interface.

The main physical mechanism governing the interface of a metal and a Mott insulator is the Kondo effect: the localized spins of the Mott insulator are screened when they are brought into contact with the metal and become therefore part of the metal. However, our numerical results show that this mechanism is not very effective as can be seen from the numerical prefactor in Eq. (4). Even for $U_{\text {right }}=U_{c}$, the quasiparticle weight is only of size $0.008 / x^{2}$. There is no small parameter in the model which controls this prefactor, which is reminiscent of another small number characterizing the physics of Mott insulators: the critical temperature describing the end-point of the first-order Mott transition is much smaller than the Mott gap both within DMFT [19] and in systems like $\mathrm{V}_{2} \mathrm{O}_{3}$ [25]. Also, the correlation length is extremely short: to obtain in Eq. (8) a correlation length of 10 lattice spacings, one has to approach the critical point with a precision of $10^{-4}$. For all practical purposes, our results imply that the Mott insulator is de facto impenetrable to the metal: Mott insulators are very good insulators and the "Kondo proximity effect" is inefficient. This is consistent with our previous study of trapped fermionic atoms in an optical lattice [8], where a metallic phase barely penetrates into a coexisting Mott insulator.

The small quasiparticle weights at $T=0$ also imply that very small temperatures larger than the local Kondo temperature, $T_{K} \propto Z$, efficiently quench the "Kondo proximity effect," see Fig. 1. Even more important is the effect of magnetism which we have neglected in our study. The tiny local Kondo temperatures in the Mott insulating phase will typically be much smaller than the exchange couplings of the spins, wiping out the Kondo effect. The magnetism of the Mott insulating phase will, in contrast, penetrate easily into the metal [18] via Friedel oscillations of the magnetization.

For the future, it will be interesting to investigate with our methods also models which are not particle-hole symmetric where interface charges and long range Coulomb interactions can lead to an electronic reconstruction of the interface [3].

We thank R. Bulla, J. Kroha, Q. Liu, T. Micklitz, M. Potthoff, and M. Vojta for useful discussions. We acknowledge supercomputer support by the John von Neumann institute for Computing (Jülich) and the
[1] W.-C. Lee and A. H. MacDonald, Phys. Rev. B 74, 075106 (2006).

[2] S. S. Kancharla and E. Dagotto, Phys. Rev. B 74, 195427 (2006).

[3] S. Okamoto and A. J. Millis, Nature (London) 428, 630 (2004); Phys. Rev. B 70, 241104(R) (2004); Phys. Rev. B 72, 235108 (2005).

[4] A. Ohtomo et al., Nature (London) 419, 378 (2002).

[5] S. Thiel, G. Hammerl, A. Schmehl, C. W. Schneider, and J. Mannhart,

Science 313, 1942 (2006).

[6] N. Reyren et al., Science 317, 1196 (2007).

[7] S. Papanikolaou et al., Phys. Rev. Lett. 100, 026408 (2008); F. Kagawa, K. Miyagawa, and K. Kanoda, Nature (London) 436, 534 (2005).

[8] R. W. Helmes, T. A. Costi, and A. Rosch, Phys. Rev. Lett. 100, 056403 (2008).

[9] T. Oka and N. Nagaosa, Phys. Rev. Lett. 95, 266403 (2005).

[10] A. Georges, G. Kotliar, W. Krauth, and M. J. Rozenberg, Reviews of Modern Physics 68, 13 (1996).

[11] W. Metzner and D. Vollhardt, Phys. Rev. Lett. 62, 324 (1989).

[12] V. Dobrosavljević and G. Kotliar, Phys. Rev. Lett. 78, 3943 (1997).

[13] M. Potthoff and W. Nolting, Phys. Rev. B 59, 2549 (1999); S. Schwieger, M. Potthoff, and W. Nolting, ibid. 67, 165408 (2003).

[14] P. Miller and J. K. Freericks, J. Phys. Condens. Matter 13, 3187 (2001); L. Chen and J. K. Freericks, Phys. Rev. B 75, 125114 (2007); J. K. Freericks, Transport in Multilayered Nanostructures (Imperial College Press, London, 2006).

[15] S. Florens, Phys. Rev. Lett. 99, 046402 (2007).

[16] M. Potthoff and W. Nolting, Phys. Rev. B 59, 2549 (1999); M. Potthoff and W. Nolting, Phys. Rev. B 60, 7834 (1999).

[17] M. P. Sarachik et al., Phys. Rev. B 58, 6692 (1998).

[18] M. Snoek et al., arXiv:cond-mat/0802.3211.

[19] R. Bulla, T. A. Costi, and D. Vollhardt, Phys. Rev. B 64, 045103 (2001).

[20] R. Bulla, T. A. Costi, and T. Pruschke, Rev. Mod. Phys. 80, 395 (2008).

[21] For the NRG [20], we use $\Lambda=2$ and 1600 kept states using the full spin symmetry.

[22] R. Bulla and M. Potthoff, Eur. Phys. J. B 13, 257 (2000).

[23] X. Y. Zhang, M. J. Rozenberg, and G. Kotliar, Phys. Rev. Lett. 70, 1666 (1993).

[24] In contrast, a Ginzburg-Landau theory reproduces exactly the mean-field theory, e.g., for an Ising model.

[25] M. Imada, A. Fujimori, and Y. Tokura, Rev. Mod. Phys. 70, 1039 (1998).

[26] For good data collapse, we have shifted the $x$ argument slightly, $x \rightarrow x-x_{0}$, where $x_{0}=-0.01$ for the DMFT + NRG results and $x_{0}=0.93$ for the Ginzburg-Landau style model for $U_{\text {left }}=2.59 D\left(x_{0}\right.$ depends on $\left.U_{\text {left }}\right)$. 